OPEN ACCESS

Edited by:

Cristina García Cáceres, Ludwig Maximilian University of

Munich, Germany

Reviewed by:

Marc Claret,

Institut de Recerca Biomèdica August

Pi i Sunyer (IDIBAPS), Spain

Kamal Rahmouni,

The University of lowa, United States

${ }^{*}$ Correspondence: Emilio Gutierrez emilio.gutierrez@usc.es

Miguel López m.lopez@usc.es

Specialty section:

This article was submitted to Neuroendocrine Science,

a section of the journal

Frontiers in Endocrinology

Received: 19 February 2021 Accepted: 15 March 2021

Published: 02 June 2021

Citation:

Fraga A, Rial-Pensado E, Nogueiras $R$,

Fernø J, Diéguez C, Gutierrez $E$ and López M (2021) Activity-Based Anorexia Induces Browning of

Adipose Tissue Independent of Hypothalamic AMPK.

Front. Endocrinol. 12:669980. doi: 10.3389/fendo.2021.669980

\section{Activity-Based Anorexia Induces Browning of Adipose Tissue Independent of Hypothalamic AMPK}

\author{
Angela Fraga ${ }^{1,2,3}$, Eva Rial-Pensado ${ }^{1,2}$, Rubén Nogueiras ${ }^{1,2}$, Johan Fernø ${ }^{4}$, \\ Carlos Diéguez ${ }^{1,2}$, Emilio Gutierrez ${ }^{3,5 *}$ and Miguel López ${ }^{1,2 *}$
}

1 Department of Physiology, Center for Research in Molecular Medicine and Chronic Diseases (CiMUS), University of Santiago de Compostela-Instituto de Investigación Sanitaria, Santiago de Compostela, Spain, ${ }^{2}$ CIBER Fisiopatología de la Obesidad y Nutrición (CIBERobn), Santiago de Compostela, Spain, ${ }^{3}$ Department of Clinical Psychology and Psychobiology, School of Psychology, University of Santiago de Compostela-Instituto de Investigación Sanitaria, Santiago de Compostela, Spain, ${ }^{4}$ Hormone Laboratory, Haukeland University Hospital, Bergen, Norway, 5 Unidad Venres Clínicos, School of Psychology, Universidad of Santiago de Compostela, Santiago de Compostela, Spain

Anorexia nervosa (AN) is an eating disorder leading to malnutrition and, ultimately, to energy wasting and cachexia. Rodents develop activity-based anorexia (ABA) when simultaneously exposed to a restricted feeding schedule and allowed free access to running wheels. These conditions lead to a life-threatening reduction in body weight, resembling AN in human patients. Here, we investigate the effect of ABA on whole body energy homeostasis at different housing temperatures. Our data show that ABA rats develop hyperactivity and hypophagia, which account for a massive body weight loss and muscle cachexia, as well as reduced uncoupling protein 1 (UCP1) expression in brown adipose tissue (BAT), but increased browning of white adipose tissue (WAT). Increased housing temperature reverses not only the hyperactivity and weight loss of animals exposed to the ABA model, but also hypothermia and loss of body and muscle mass. Notably, despite the major metabolic impact of ABA, none of the changes observed are associated to changes in key hypothalamic pathways modulating energy metabolism, such as AMP-activated protein kinase (AMPK) or endoplasmic reticulum (ER) stress. Overall, this evidence indicates that although temperature control may account for an improvement of AN, key hypothalamic pathways regulating thermogenesis, such as AMPK and ER stress, are unlikely involved in later stages of the pathophysiology of this devastating disease.

Keywords: activity-based anorexia, temperature, cachexia, brown adipose tissue, white adipose tissue, hypothalamus, AMPK, ER stress

\section{INTRODUCTION}

Anorexia nervosa (AN) is an eating disorder characterized by decreased food intake, severe weight loss and hyperactivity $(1,2)$. Due to chronic underfeeding, patients with AN present neuroendocrine changes, in an attempt to adapt to malnutrition, which in many cases are not completely reversed even with the recovery of body weight (3); this leads to several medical complications $(4,5)$. 
Activity-Based Anorexia (ABA) is considered the best analogue animal model for AN (6), which is obtained by providing availability of food to rats 1-2 $\mathrm{h}$ /day and free access to a running wheel (7). Under these circumstances, rats develop an excessive running and reduced meal efficiency, eliciting massive weight loss and hypothermia, both mimicking the principal signs of AN disorder in humans. Notably, ABA also reproduces the metabolic and endocrine abnormalities observed in humans (8). AN-associated hyperactivity has been proposed as an adaptative behavioral response to compensate for hypothermia (9). Previous research has shown that exposure to a high ambient temperature (AT) prevents and reverses the hyperactivity and improves feeding patterns, allowing body weight recovery in both male and female rats under ABA conditions (10-15). These beneficial effects of temperature have been also found in the semi-starvation induced hyperactivity model (SIH) (16).

Due to the ability of the ABA model to reproduce many of the symptoms of the AN disorder in humans, as well as the identification of several genes involved in food intake regulation and energy balance as potential pathways that contribute to the etiology and maintenance of $\operatorname{AN}(17,18)$, it would be interesting to examine the effect of high AT on energy sensors potentially involved in $\mathrm{AN}$, as well as the possible clinical implications on the treatment of AN in humans. Here, we focused on AMP-activated protein kinase (AMPK) and endoplasmic reticulum (ER) stress, well-known mechanisms regulating both sides of the energy metabolism, namely feeding and thermogenesis (19-26).

\section{MATERIALS AND METHOD}

\section{Animals}

Male Sprague-Dawley rats (130-190 g) were acquired from the Animalario General USC, (Santiago de Compostela, Spain). They were kept with food and water ad libitum on a 12-hr light-dark cycle (LD, lights on from 08:00 to 20:00 hours). Ambient temperature set at $21 \pm 1^{\circ} \mathrm{C}$. The Ethics Committee on the use and care of animals of Santiago de Compostela University approved all described procedures (project license 15004/17/ 002). All experiments were carried out in accordance with Royal Decree 53/2013 of February 1, Law 32/2007 of November 7, and European Communities Council Directive 2010/63/UE of September 22, on the protection of animals used for experimental and other scientific purposes.

\section{Running Wheels}

Cages $(48 \times 31.5 \times 47 \mathrm{~cm})$ equipped with a Whatman-type activity wheel $(1.12-\mathrm{m}$ circumference $35.7 \mathrm{~cm}$ diameter, $10-\mathrm{cm}$ wide running surface of a $10-\mathrm{mm}$ mesh bounded by clear Plexiglas and stainless-steel walls; Panlab Harvard Apparatus; Barcelona, Spain) were placed inside wooden incubators $(60 \times 60 \times$ $60 \mathrm{~cm}$ ) with polycarbonate roofs, provided with a $150 \mathrm{~W}$ heat wave lamp, connected to a thermostat and a probe positioned at the level of the animal, which allowed individual control of AT.

\section{ABA Procedure}

One week prior to the start of the experiments body temperature and activity transmitters (PTD 4000 E-Mitter, Respironics Mini Mitter Inc; Bend, OR, US) were implanted under ketaminexylazine anesthesia (50 mg/kg, intraperitoneal) and inserted in a subcutaneous pocket on the ventral surface created using blunt dissection. The rats were allowed seven days to recover. On the eighth day, rats were weighed and assigned to two weight matched groups: an active and restrictive-fed (AC) group and an inactive and restrictive-fed (IN) group. All the rats were transferred to running wheel cages, but only the rats assigned to the active condition had access to functional wheels. The rats assigned to the inactive condition remained with the activity wheel blocked during the whole experiment, avoiding any possibility of movement inside those devices. The ABA procedure started (day 0 ) with the removal of food at 12:30 h for restricted-fed groups. At the same time, the doors to the wheels were opened for the active group. From day 1 onward, all rats were given access to food according to a restricted feeding schedule from 11:00 to 12:30 h. The doors of the wheels were closed during this feeding period. Food intake was measured by weighing the food at the beginning and the end of every $1.5 \mathrm{~h}$ feeding period. Rats were also weighed daily at 10:30 h (as they were on day 0 ). This phase continued for each restricted-fed active rats until it reached a body weight loss criterion (BWLC) of $20 \%$ of their day 0 body weight. At this time, rats were assigned to one of two ambient temperature, $21^{\circ} \mathrm{C}$ or $32^{\circ} \mathrm{C}$, as indicated in the two digits of the abbreviated group name (AC21 and AC32). These conditions were maintained until rats reached either the recovery criterion, which was defined as body weight on any particular day (day $n$ ) greater than the weight of the animal 4 days before, (day $n-4$ ), or the removal criterion which was defined as body weight under $75 \%$ of body weight on day 0 (7). The experiment was terminated after 15 days. The restrictedfed inactive rats were also assigned to two ambient temperature conditions, $21^{\circ} \mathrm{C}$ or $32^{\circ} \mathrm{C}$ (IN21 and IN32). For the rats maintained at $21^{\circ} \mathrm{C}$ the experiment lasted only six days (median of days that the restricted-fed active animals took to reach the $\mathrm{BWLC})$. While rats assigned to $21^{\circ} \mathrm{C} \mathrm{AT}$ remained in these conditions for three days more (median of days that de AC21 group took to reach the removal criterion), rats assigned to $32^{\circ} \mathrm{C}$ remained six days on experiment (median of days that the AC32 group took to reach the recovery criterion). At the end of the experiments rats were sacrificed by decapitation after the weighing routine; trunk blood, brain, brown adipose tissue (BAT), hind leg muscle and gonadal white adipose tissue (gWAT) were collected, frozen and stored at $-80^{\circ} \mathrm{C}$ until assay.

\section{Blood Biochemistry}

Trunk blood was collected into specific tubes (BD Vacutainer; Plymouth, UK) and centrifuged at $3,200 \mathrm{x}$ g for $15 \mathrm{~min}$ at $4^{\circ} \mathrm{C}$ to separate the serum. Then serum was stored at $-80^{\circ} \mathrm{C}$. Glucose free T3 and free T4 were measured using an automated chemistry analyzer (ADVIA 2400 Chemistry System, Siemens Medical Solutions Inc; Ann Arbor, MI US). Leptin, corticosterone (CORT), adrenaline and noradrenaline levels were measured 
using ELISA kits (EZRL-83K; Linco Research; St. Charles, Missouri, US, for leptin; ab108821, Abcam, Cambridge, UK, for CORT; EIA-3175; DRG Instruments GmbH, Marburg, Germany, for adrenaline and noradrenaline).

\section{Hypothalamic Dissection}

The brain was placed in an adult rat brain matrix (Kent-Scientific Corporation, \#RBMA-300C; Torrington, CT, US) with the hypothalamus upward and dissected as previously described $(23,24,26-28)$.

\section{Western Blotting}

BAT, gWAT and the hypothalamic nuclei (arcuate, ARC, and ventromedial, VMH) were homogenized in lysis buffer containing protease inhibitor cocktail tablets (Roche Diagnostics; Indianapolis, IN, US) and the protein concentration was determined using the Bradford method (Protein assay dye concentrate, Bio-Rad Laboratories; Hercules, CA, US). The protein lysates were subjected to SDS-PAGE and electro-transferred to polyvinylidene difluoride membranes (PVDF; Millipore; Billerica, MA, US) with a semidry blotter. Membranes were blocked in TBS/Tween with 3\% of BSA (Bovine serum albumin, Sigma Aldrich, St. Louis, US) and probed with the following antibodies against: pAMPK $\alpha$ (Thr172), glucoseregulated protein 78 (GRP78; Cell Signaling; Danvers; MA, US), UCP1 (uncoupling protein 1; Abcam; Cambridge, UK), C/EBP Homologous Protein (CHOP; SCBT; Dallas, Texas, USA), $\alpha$-tubulin and $\beta$-actin (Sigma-Aldrich; St. Louis, MO, US), as previously shown $(23,24,26-29)$. Membranes were incubated with the corresponding secondary antibody: anti-rabbit, antimouse, or anti-goat (DAKO; Glostrup, Denmark). Detection of proteins was performed with Enhanced chemiluminescence (ECL) reagents (Pierce ECL Western Blotting Substrate, Cultek; Madrid, Spain) according to the manufacturer's instructions, exposed to x-ray films (Fujifilm; Tokyo, Japan), developed and fixed under appropriate dark room conditions. Autoradiographic films were scanned and the bands signal was quantified by densitometry using ImageJ-1.33 software (NIH; Bethesda, MD, US), as shown $(23,24,26-29)$. Values were expressed in relation to $\beta$-actin (hypothalamus) or $\alpha$-tubulin (BAT). Representative images for all proteins are shown, all the bands for each picture always come from the same gel, although they may be spliced for clarity, as represented by vertical lines.

\section{Real-Time Quantitative RT-PCR}

Real-time PCR (TaqMan ${ }^{\circledR}$; Applied Biosystems; Foster City, CA, USA) was performed using specific primers and probes (Supplementary Table 1), as shown (27-30). Values were expressed in relation to hypoxanthine-guanine phosphoribosyltransferase (Hprt) levels.

\section{Hematoxylin-Eosin Staining and UCP1 Immunohistochemistry}

gWAT depots were fixed in $10 \%$ buffered formaldehyde and subsequently treated for histological study by dehydration (increasing alcohol concentrations), mounting in xylene and immersion in paraffin. The paraffin blocks were sliced into 3 $\mathrm{mm}$ sections that were processed, deparaffinized in xylene, rehydrated and rinsed in distilled water and then stained either for hematoxylin-eosin or UCP1 immunohistochemistry. For the hematoxylin-eosin processing, slices were first stained with hematoxylin for $5 \mathrm{~min}$, washed and stained again with eosin for $1 \mathrm{~min}$. For UCP1 immunohistochemistry, slices were incubated overnight with the primary antibody (UCP1; Abcam; Cambridge, UK), washed and incubated with the secondary antibody (DAKO; Glostrup, Denmark). Images were taken in an optical microscope with a digital camera Olympus XC50 (Olympus Corporation; Tokyo, Japan) at 40X. Adipocyte area and UCP1 staining area were quantified using ImageJ 1.33 software (NIH, Bethesda, MD, US), as shown $(25,29,31,32)$.

\section{Statistical Analysis}

Data are presented as mean \pm SEM. When two groups were compared, statistical significance was determined by two-sided Student's t-test; when more than groups were compared, statistical significance was determined by ANOVA followed by Bonferroni's test. $\mathrm{P}<0.05$ was considered significant. Statistical analyses were performed using SPSS 21.0 software (IBM; Armonk, NY, US).

\section{RESULTS}

\section{Increased Housing Temperature Reverses the Effect of ABA on Energy Balance and Activity}

ABA rats exposed at a housing temperature of $32^{\circ} \mathrm{C}(\mathrm{AC} 32)$ ran six-fold less than rats housed at $21^{\circ} \mathrm{C}$ (AC21). During Phase II running activity of $\mathrm{AC} 32$ rats did not reach the activity level shown on the last day of Phase I, (day they met the body weight loss criterion of $20 \%, 20 \%$ BWLC), despite being an average of 4 more days being subjected to standard ABA conditions (restricted feeding plus wheel access) (Figure 1A). Active rats lose weight during Phase II while inactive rats keep it stable. Besides, the increase in room temperature to $32^{\circ} \mathrm{C}$ allowed for less weight loss in active rats and slightly increased weight in inactive rats, when compared to their counterparts at $21^{\circ} \mathrm{C}$ (Figures 1B, C). Both active and inactive rats exhibited higher body temperature when they were maintained at $32^{\circ} \mathrm{C}$, as (Figures 1D, E). Rats kept at lower temperature of $21^{\circ} \mathrm{C}$ initially ate more than rats at $32^{\circ} \mathrm{C}$ although no significant differences were detected on the final day (Figures 1F, G).

\section{Increased Housing Temperature Reverses the Effects of ABA on Circulating Parameters}

Next, we evaluated the effect of housing temperature on circulating parameters in the ABA model (Table 1). We first focused on leptin levels, since this hormone has been shown to have a controversial role in this model of disease (16, 33-39). Resembling the clinical evidence $(34,36,37)$, our data showed that active rats had significantly lower circulating leptin levels that inactive ones, which were elevated after exposure of the rats 


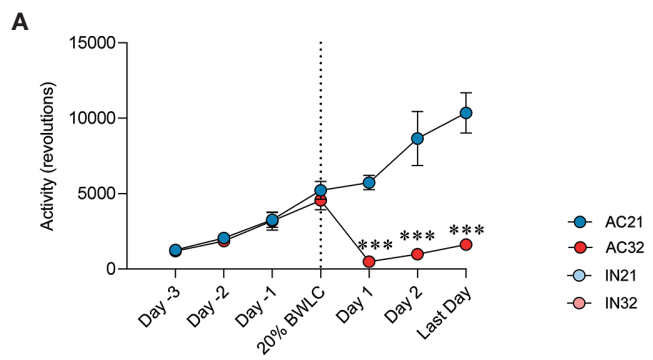

B

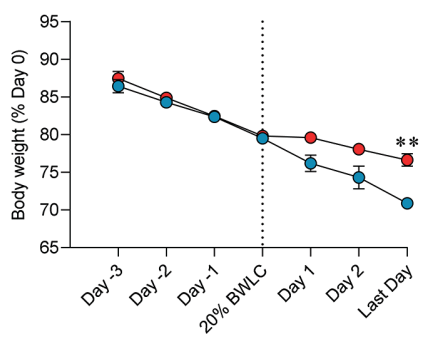

D

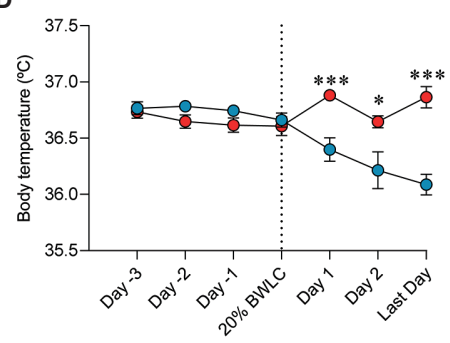

$\mathbf{F}$

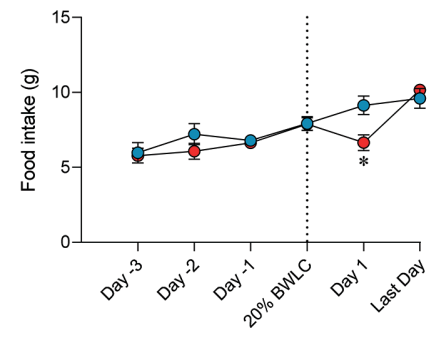

C

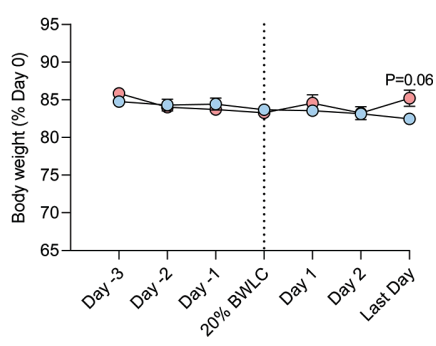

E

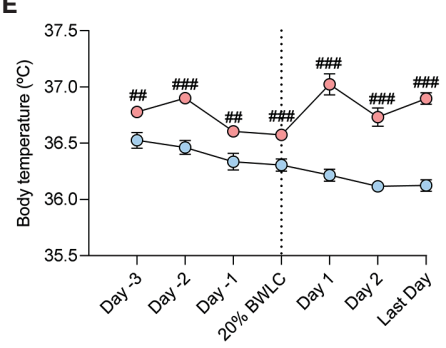

G

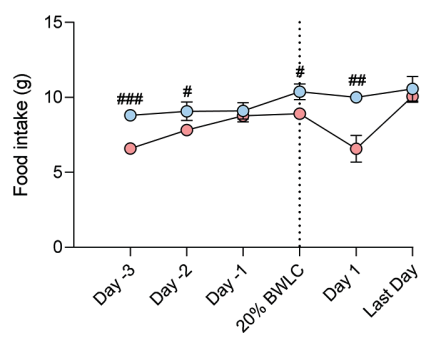

FIGURE 1 | Effect of ABA and temperature on energy balance. (A) Activity ( $n=9-12$ rats/group) (B, C) Body weight (\% of day 0) ( $n=8-12$ rats/group) (D, E) Body temperature $\left(\mathrm{n}=7-10\right.$ rats/group) $(\mathbf{F}, \mathbf{G})$ Food intake $\left(\mathrm{n}=8-12\right.$ rats/group) of active rats at $21^{\circ} \mathrm{C}$ and $32^{\circ} \mathrm{C}(\mathrm{AC} 21$ and $\mathrm{AC} 32)$ and inactive rats at $21^{\circ} \mathrm{C}$ and $32^{\circ} \mathrm{C}$

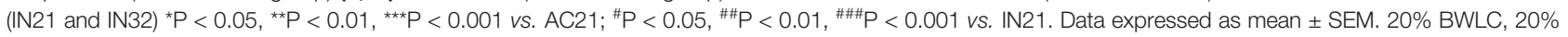
body weight loss criterion.

to $32^{\circ} \mathrm{C}$ (Table 1). No major changes were detected in glycaemia. Regarding CORT, active rats housed at $21^{\circ} \mathrm{C}$ displayed the highest circulating levels of this hormone (Table 1), similarly to AN patients and other preclinical models (40-44), indicating greater stress. Notably, when maintained at $32^{\circ} \mathrm{C}$, active rats normalized their circulating CORT, reaching even lower concentration than the inactive groups (Table 1). Thyroid hormones (T4 and T3) play a major role in the modulation of temperature $(45,46)$, and we investigated how their circulating levels were affected in our setting. Inactive rats displayed the expected correlation between ambient temperature and thyroid status. Interestingly, that effect was not evident in active rats, which showed lower T4 and T3 when kept at $21^{\circ} \mathrm{C}$ as compared to $32^{\circ} \mathrm{C}$ (Table 1). Finally, active rats at $21^{\circ} \mathrm{C}$ also showed higher levels of noradrenaline, that were reduced when housed at $32^{\circ} \mathrm{C}$ (Table 1). 
TABLE 1 | Serum parameters in the experimental groups.

\begin{tabular}{|c|c|c|c|c|}
\hline & Active $21^{\circ} \mathrm{C}$ & Active $32^{\circ} \mathrm{C}$ & Inactive $21^{\circ} \mathrm{C}$ & Inactive $32^{\circ} \mathrm{C}$ \\
\hline Leptin (ng/mL) & $0.20 \pm 0.002 ! ! !$ & $0.24 \pm 0.005^{\star \star \star}$ & $0.28 \pm 0.02$ & $0.29 \pm 0.02$ \\
\hline Glucose (mg/dL) & $120.33 \pm 8.29$ & $137.75 \pm 3.28^{\star}$ & $129.86 \pm 5.82$ & $140.00 \pm 3.49$ \\
\hline Corticosterone (ng/mL) & $1466.87 \pm 125.28 ! ! !$ & $273.05 \pm 43.77^{\star \star \star}$ & $412.98 \pm 63.40$ & $435.91 \pm 35.57$ \\
\hline $\mathrm{T} 4$ (ng/dL) & $0.98 \pm 0.05 ! ! !$ & $1.54 \pm 0.07^{\star \star \star}$ & $1.89 \pm 0.07$ & $1.71 \pm 0.04^{\#}$ \\
\hline T3 (pg/mL) & $1.51 \pm 0.09 ! ! !$ & $2.24 \pm 0.05^{\star \star \star}$ & $3.68 \pm 0.05$ & $3.07 \pm 0.09^{\# \# \#}$ \\
\hline Adrenaline (ng/mL) & $2.46 \pm 0.50$ & $2.16 \pm 0.19$ & $4.18 \pm 1.21$ & $3.43 \pm 0.77$ \\
\hline Noradrenaline (ng/mL) & $2.64 \pm 0.32$ & $1.75 \pm 0.17^{\star}$ & $3.54 \pm 0.58$ & $3.58 \pm 0.58$ \\
\hline
\end{tabular}

$n=9-11$ animals/group.

${ }^{*} P<0.05$ and ${ }^{* *} P<0.001$ vs. AC21.

${ }^{\#} P<0.05$, and ${ }^{\# \# \#} P<0.001$ vs. IN21.

!!! $P<0.001$ IN21 vs. AC21.

\section{Increased Housing Temperature Reverses the Effects of ABA on WAT and Skeletal Muscle}

$\mathrm{AN}$ is also characterized by a great loss of fat mass $(38,47,48)$. Therefore, we decided to explore lipogenesis and lipolysis markers in the WAT of ABA rats. Active rats at $21^{\circ} \mathrm{C}$ had an extreme decrease in all examined lipogenic markers levels, such as acetyl-CoA carboxylate $\alpha$ (ACC $\alpha$ ), fatty acid synthase (FAS), sterol regulatory element-binding protein 1 (SREBP1), peroxisome proliferator-activated receptor-gamma (PPAR $\gamma$ ) and CCAAT/enhancer binding protein alpha and beta $(\mathrm{C} /$ $\mathrm{EBP} \alpha$ and $\mathrm{C} / \mathrm{EBP} \beta)$, compared to inactive rats. Active rats at $32^{\circ} \mathrm{C}$ showed a marked recovery in the expression of these factors (Figure 2A). Increased housing temperature did not impact on

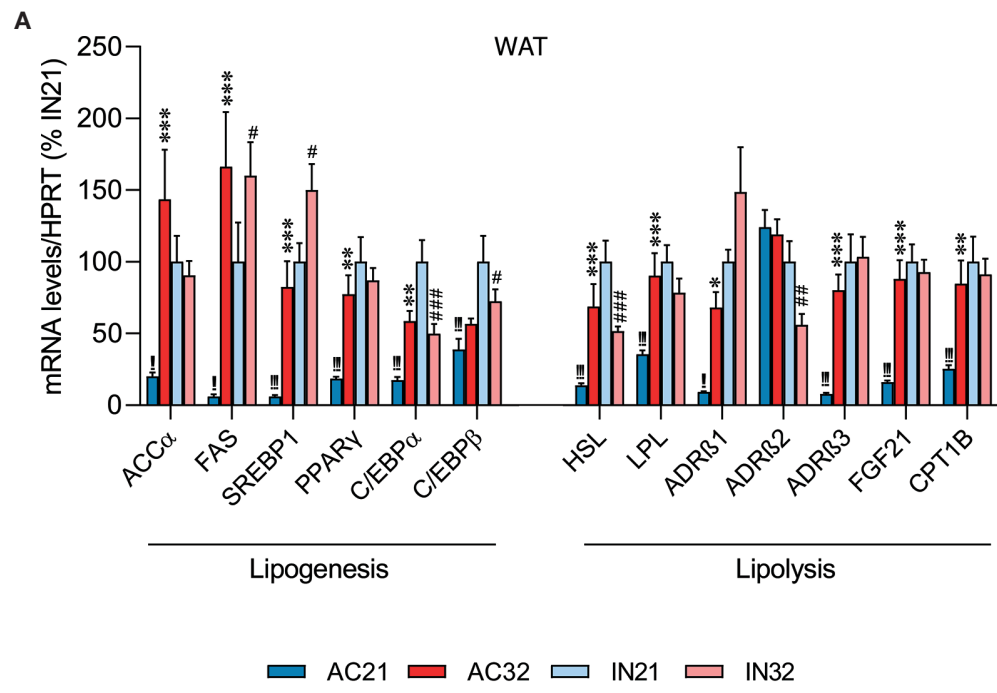

B

Skeletal muscle

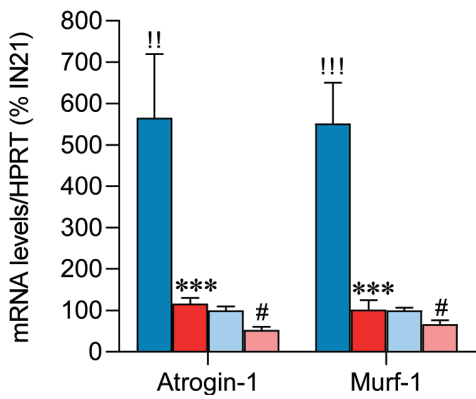

FIGURE 2 | Effect of ABA and temperature on WAT and muscle. mRNA levels of (A) lipogenesis and lipolysis markers in the WAT ( $\mathrm{n}=8-11$ rats/group) and (B) cachexia markers in skeletal muscle $\left(\mathrm{n}=8-11\right.$ rats/group) of active rats at $21^{\circ} \mathrm{C}$ and $32^{\circ} \mathrm{C}(\mathrm{AC} 21$ and $\mathrm{AC} 32)$ and inactive rats at $21^{\circ} \mathrm{C}$ and $32^{\circ} \mathrm{C}$ (IN21 and IN32). ${ }^{*} \mathrm{P}<0.05,{ }^{* *} \mathrm{P}<0.01,{ }^{* \star} \mathrm{P}<0.001$ vs. AC21; ${ }^{\#} \mathrm{P}<0.05,{ }^{\# \#} \mathrm{P}<0.01,{ }^{\# \# \#} \mathrm{P}<0.001$ vs. IN21; "'P $<0.01,{ }^{\text {!"! } P}<0.001$ IN21 vs. AC21. Data expressed as mean $\pm \mathrm{SEM}$. 
gene expression levels in inactive rats, except for an increase in FAS and SREBP1 mRNA expression (Figure 2A). On the other hand, the levels of lipolysis markers, such as hormone-sensitive lipase (HSL), lipoprotein lipase (LPL), adrenergic receptor beta 1 $(\mathrm{ADR} \beta 1)$ and adrenergic receptor beta $3(\mathrm{ADR} \beta 3)$, but not beta 2 (ADR 32 ), fibroblast growth factor 21 (FGF21) and carnitine palmitoyltransferase $1 \mathrm{~B}(\mathrm{CPT} 1 \mathrm{~B})$, were reduced in active rats at $21^{\circ} \mathrm{C}$, likely due to the massive loss of adiposity of these animals, while heat reversed this expression (Figure 2A).

AN patients have a reduction in lean mass and wasting syndrome, leading to cachexia $(3,49,50)$. Therefore, we explored two cachexia markers in skeletal muscle, namely Atrogin-1 and Murf-1. Our data showed that rats housed at $21^{\circ} \mathrm{C}$ exhibited a markedly increased expression of cachectic markers relative to rats housed at $32^{\circ} \mathrm{C}$, evident both in the inactive and the active cohort, possibly indicating muscle deteriorating (Figure 2B).

\section{ABA Reduces BAT UCP1 Levels But Increases the Browning of WAT}

It is known that $\mathrm{AN}$ is associated with impaired thermogenesis (50). In fact, it has been reported that young women with AN exhibit reduced cold-activated BAT (50). Analysis of UCP1 expression in the BAT of our model showed decreased $21^{\circ} \mathrm{C}$ induced UCP1 protein levels in AC rats (Figures 3A, B). As expected, increased environmental temperature decreased UCP1 expression in both active and inactive animals (Figures 3A, B).

Over the last years, accumulating evidence have demonstrated that activation of beige/brite ("brown in white") adipocytes in the WAT, a process known as browning (51-53), is responsible for a
A

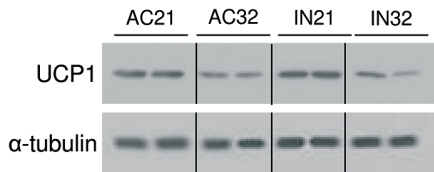

WAT

C

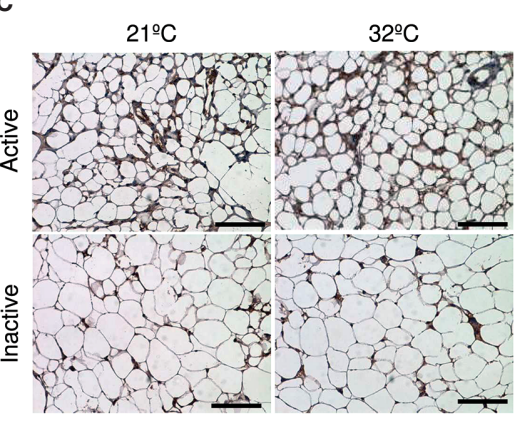

E

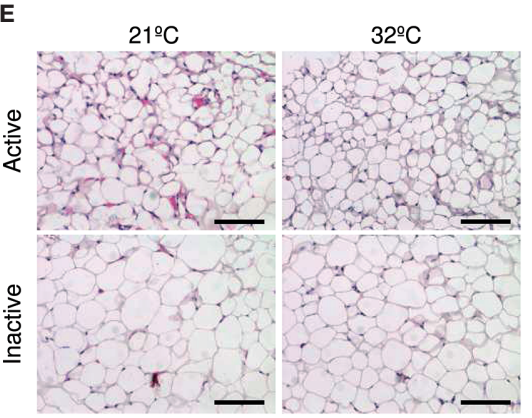

$B$
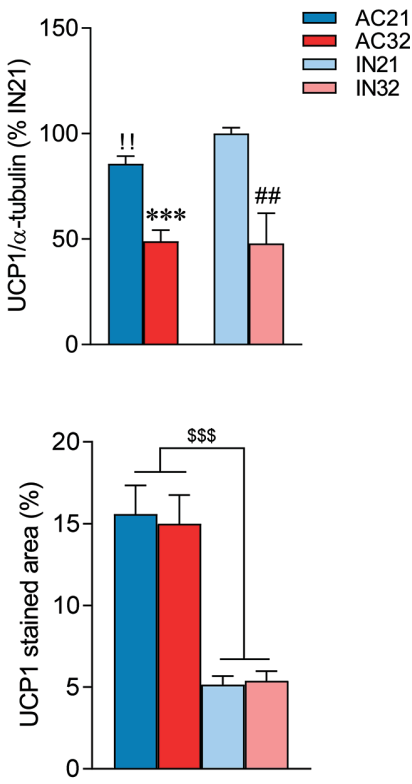

$\mathbf{F}$

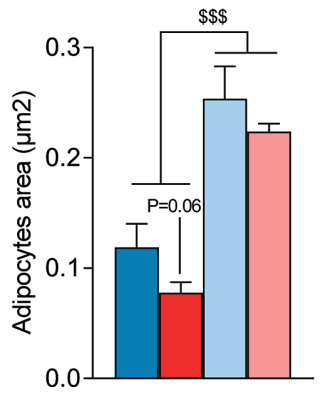

FIGURE 3 | Effect of ABA and temperature on BAT and WAT browning. (A, B) Protein levels of UCP1 in the BAT ( $\mathrm{n}=7$ rats/group) (C, D) UCP1 staining in WAT $(\mathrm{n}=8-12$ rats/group) (E, $\mathbf{F})$ Adipocyte are in WAT ( $\mathrm{n}=8-12$ rats/group) of active rats at $21^{\circ} \mathrm{C}$ and $32^{\circ} \mathrm{C}(\mathrm{AC} 21$ and $\mathrm{AC} 32)$ and inactive rats at $21^{\circ} \mathrm{C}$ and $32^{\circ} \mathrm{C}(\mathrm{IN} 21$ and IN32). ${ }^{* * * P}<0.001$ vs. AC21; ${ }^{\# \# P}<0.01$ vs. IN21; " $\mathrm{P}<0.01$ IN21 vs. AC21; ${ }^{\$ \$ \$} \mathrm{P}<0.001$ for simplification. Data expressed as mean \pm SEM. The bands in gels from panel (A) have been spliced from the same original gels. Scale bar: $100 \mu \mathrm{m}$. 
significant increase in total energy expenditure (54). Notably, recent studies have also linked the browning of WAT to other wasting syndromes, such as cancer-induced cachexia $(55,56)$; however, to date, no data have linked AN to browning of WAT. Our histological analysis of WAT showed that ABA rats exhibited a "brown-like" multilocular pattern, associated with increased UCP1 immunostaining (Figures 3C, D) and decreased adipocyte area (Figures 3E, F). Importantly, the induction of browning was not affected by housing temperature (Figures 3C-F). Overall, these data indicate that $\mathrm{ABA}$ rats, besides hypophagia, also displayed increased browning of WAT, that was compatible with the elevated catabolic state.

\section{ABA Does Not Impact Either AMPK or ER Stress in the Hypothalamus}

Finally, we aimed to investigate if $\mathrm{ABA}$ might result in changes at the central level that could explain the catabolic state of this model. One of the principal regulators of energy balance at a central level is hypothalamic AMPK, an energy sensor that controls both sides of the energy balance equation: food intake and energy expenditure (20-22). Firstly, we investigated the effect of $\mathrm{ABA}$ and temperature on total hypothalamic extracts; our data did not show any significant impact of either ABA or temperature on the protein levels of the AMPK signaling pathway (Supplementary Figure 1A). Current data indicate that the effects of AMPK in the hypothalamus are nucleusspecific; thus while AMPK in the ARC is mainly involved in the regulation of feeding, AMPK in the $\mathrm{VMH}$ regulates $\mathrm{BAT}$ thermogenesis and browning of WAT $(19-23,32)$, Therefore, we performed further analysis of AMPK in ARC and VMH enriched protein lysates, which showed a non-significant tendency of phosphorylated AMPK (pAMPK) to be increased in the $\mathrm{VMH}$ of $\mathrm{ABA}$ rats, that might account for the decreased levels of BAT UCP1 protein levels observed in those animals (Figures 4A, B). No major effect of housing temperature was detected of pAMPK levels in the VMH (Figures 4A, B). Similar data were found when pAMPK was assayed in the ARC (Figures 4C, D). Overall, these results indicated that the impaired feeding and browning that characterized ABA model were unlikely associated to changes in AMPK signaling in these nuclei.

Finally, we investigated the effect of ABA on hypothalamic ER stress signaling, since recent data have linked this cellular response with the regulation of BAT thermogenesis and browning of WAT $(24-26,28)$. Our data did not show any major impact of either ABA or housing temperature on two key hypothalamic ER stress markers, namely GRP78 and CHOP (Figures 4E, F), excluding their association in the BAT and metabolic alterations of this model.

\section{DISCUSSION}

Here, we show that the catabolic state that characterizes ABA is associated with major changes in BAT thermogenesis and WAT browning. Notably, those changes are not related to modification in key central regulators of adipose tissue activity, namely hypothalamic AMPK and ER stress signaling.

AN is characterized by energy balance impairment as a result of decreased food intake and hyperactivity, leading to severe weight loss $(1,2)$. Different brain regions, such as rewardmotivated learning or hippocampal structures, have been involved in the pathology of $\mathrm{AN}(1,2,8,57)$. Here, we aimed to investigate whether the canonical hypothalamic (VMH)AMPK-ER stress-SNS-BAT axis $(21,22)$ could be involved in the reduced feeding and the changes in BAT and WAT browning that characterize the $\mathrm{ABA}$ model in rats.

$\mathrm{ABA}$ is considered the best analogue animal model for AN (6). In addition, it is well-established that ABA-induced hyperactivity is an adaptative behavioral response to compensate for hypothermia (9). Our data are in line with previous studies reporting the beneficial effect of increased ambient temperature to $32^{\circ} \mathrm{C}$ on the recovery of rats subjected to the ABA model, even after $20 \%$ weight loss has occurred $(10-13,15,58)$. Although our data confirm former evidence, the use of temperature recording by telemetry allows a constant monitoring of the body temperature throughout the experiment, which constitutes a big advantage when compared to previous reports. Food-restricted rats suffered hypothermia when given free access to a running wheel, as body temperature decreases over days. On the contrary, rats exposed to $32^{\circ} \mathrm{C}$, both active and inactive, avoid hypothermia and their body temperature at the end of the experiment reached values higher than when meeting the weight loss criterion. These findings reinforce the hypothesis that hyperactivity is an adaptive response to compensate for the hypothermia derived from weight loss (9).

In mammals, the BAT is responsible for the adaptative thermogenesis which regulates body temperature when other mechanisms (i.e., heat conservation) are not enough to maintain homeothermy $(59,60)$. Food-restriction elicits reductions in energy expenditure through decreased BAT thermogenesis, as a strategy to save energy, although it leads to a hypothermic state (61-63). In ABA rats this response is exacerbated, entering in a vicious cycle situation that potentiates an overall catabolic state leading to wasting and cachexia. Notably, the increase in housing temperature reduced the expression of UCP1 in the BAT. That reduction in adaptive thermogenesis together with the reduction of hyperactivity would account for a better preservation of body mass (16) and the recovery of body weight of rats exposed to the ABA model. This is also demonstrated by an improved metabolic profile at the higher ambient temperature, exemplified by the reduction in the expression of cachectic markers in skeletal muscle and the increased WAT lipogenesis. Still, the recovery in body mass is not total, likely due to the maintained browning of WAT, which may account for a chronic increased energy expenditure (54), leading to a sustained basal catabolic state.

There are a huge amount of data linking hypothalamic AMPK and ER stress pathways in the hypothalamus, specifically in the $\mathrm{VMH}$, with the regulation of thermogenesis in BAT, as well as the browning of WAT (19-26). This prompted us to investigate whether those hypothalamic molecular mediators could be associated to the BAT and WAT responses in the ABA model. 


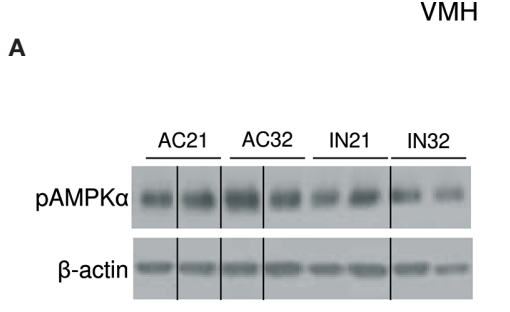

ARC
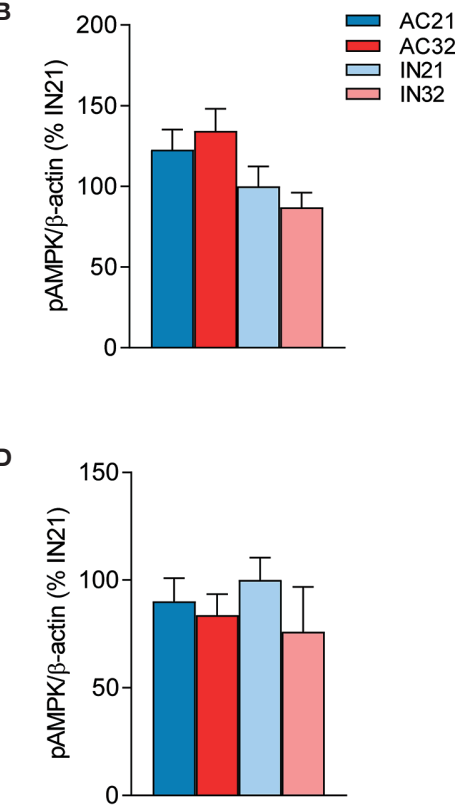

$\mathrm{VMH}$
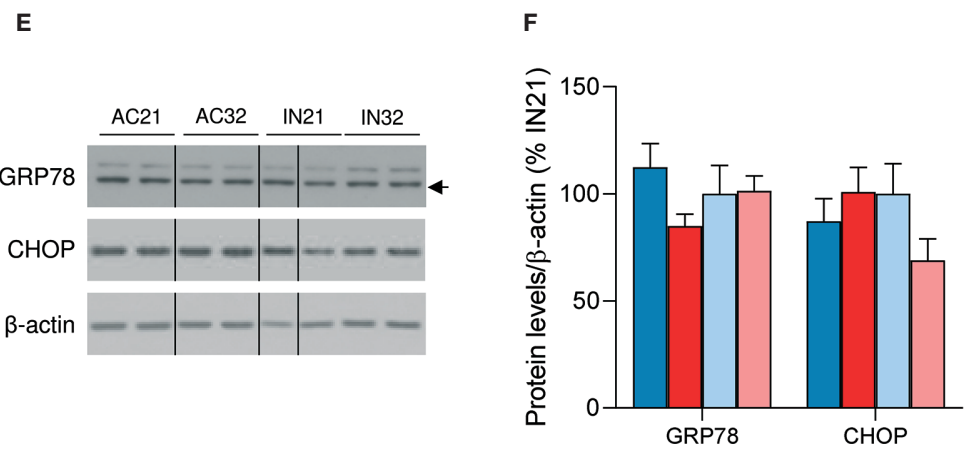

FIGURE 4 | Effect of ABA and temperature on AMPK and ER stress in the VMH and ARC. (A, B) Protein levels of pAMPK $\alpha$ in the VMH ( $n=7-10$ rats/group) (C, D) Protein levels of pAMPK $\alpha$ in the ARC ( $n=7$ rats/group) (E, F) Protein levels of GRP78 and $\mathrm{CHOP}$ in the VMH ( $\mathrm{n}=7$ rats/group) of active rats at $21^{\circ} \mathrm{C}$ and $32^{\circ} \mathrm{C}$ (AC21 and AC32) and inactive rats at $21^{\circ} \mathrm{C}$ and $32^{\circ} \mathrm{C}$ (IN21 and IN32). Data expressed as mean \pm SEM. The bands in gels from panel (A, C, E) have been spliced from the same original gels.

Our analysis did not find major expression differences in the levels of pAMPK (the active isoform), GRP78 and CHOP either in the $\mathrm{VMH}$ and/or the ARC of ABA rats. In fact, this result is opposite to a recent report where it has been described that hypothalamic pAMPK levels are reduced in ABA mice (64). These discrepancies could be likely explained by the different species (rats vs. mice), but also by the nuclei-specific an analysis performed in our study, which is critical to understand AMPK and ER stress function in the hypothalamus (19-26) at the studied times. Moreover, timing could be also a factor, in this sense it is likely that at the final time point that we investigated, initial changes in hypothalamic AMPK and/or ER stress (maybe responsible for the BAT and browning changes observed) could not be detected. Further work will be needed to address the exact role of these molecular mechanisms in the pathology of AN.

In summary, our study shows a general description of the metabolic state of rats exposed to the ABA model and of those rats treated with heat. The results are consistent with the hypothesis that body temperature is an important parameter in ABA. The application of heat reverses not only the hyperactivity and weight loss of animals exposed to the ABA model, but also hypothermia, hypoleptinemia and loss of muscle mass. However, none of the changes observed are associated to changes in key hypothalamic pathways modulating energy metabolism, such as AMPK or ER stress (19-26) at the studied times. Hence, hypothermia in AN should be given more attention in future 
research to study the underlying brain mechanism involved in the warming effect and to explore new treatments.

\section{DATA AVAILABILITY STATEMENT}

The raw data supporting the conclusions of this article will be made available by the authors, without undue reservation.

\section{ETHICS STATEMENT}

The animal study was reviewed and approved by The Ethics Committee on the use and care of animals of Santiago de Compostela University approved all described procedures (project license 15004/17/002). All experiments were carried out in accordance with Royal Decree 53/2013 of February 1, Law 32/2007 of November 7, and European Communities Council Directive 2010/63/UE of September 22, on the protection of animals used for experimental and other scientific purposes.

\section{AUTHOR CONTRIBUTIONS}

$\mathrm{AF}$ and ER-P performed the in vivo experiments, analytical methods and collected the data. AF, EG and ML designed the experiments and analyzed the data. AF, ER-P, RN, JF, CD, EG and ML interpreted and discussed the data. AF, EG and ML developed the hypothesis. AF and ML made the figures and

\section{REFERENCES}

1. Treasure J, Zipfel S, Micali N, Wade T, Stice E, Claudino A, et al. Anorexia Nervosa. Nat Rev Dis Primers (2015) 1:15074. doi: 10.1038/nrdp.2015.74

2. Frank GKW, Shott ME, DeGuzman MC. The Neurobiology of Eating Disorders. Child Adolesc Psychiatr Clin N Am (2019) 28(4):629-40. doi: 10.1016/j.chc.2019.05.007

3. Singhal V, Misra M, Klibanski A. Endocrinology of Anorexia Nervosa in Young People: Recent Insights. Curr Opin Endocrinol Diabetes Obes (2014) 21 (1):64-70. doi: 10.1097/MED.0000000000000026

4. Gibson D, Workman C, Mehler PS. Medical Complications of Anorexia Nervosa and Bulimia Nervosa. Psychiatr Clin North Am (2019) 42(2):263-74. doi: $10.1016 /$ j.psc.2019.01.009

5. Schorr M, Miller KK. The Endocrine Manifestations of Anorexia Nervosa: Mechanisms and Management. Nat Rev Endocrinol (2017) 13(3):174-86. doi: 10.1038/nrendo.2016.175

6. Gutierrez E. A Rat in the Labyrinth of Anorexia Nervosa: Contributions of the Activity-Based Anorexia Rodent Model to the Understanding of Anorexia Nervosa. Int J Eat Disord (2013) 46(4):289-301. doi: 10.1002/eat.22095

7. Routtenberg A, Kuznesof AW. Self-Starvation of Rats Living in Activity Wheels on a Restricted Feeding Schedule. J Comp Physiol Psychol (1967) 64 (3):414-21. doi: 10.1037/h0025205

8. Schalla MA, Stengel A. Activity Based Anorexia as an Animal Model for Anorexia Nervosa-a Systematic Review. Front Nutr (2019) 6:69. doi: 10.3389/ fnut.2019.00069

9. Gutierrez E, Vazquez R, Boakes RA. Activity-Based Anorexia: Ambient Temperature Has Been a Neglected Factor. Psychon Bull Rev (2002) 9 (2):239-49. doi: 10.3758/BF03196278 wrote the manuscript. ML is the senior author and lead contact, secured funding, coordinated, and led the project. All authors contributed to the article and approved the submitted version.

\section{FUNDING}

The research leading to these results has received funding from the Xunta de Galicia (RN: 2016-PG057; ML: 2016-PG068); Ministerio de Economía y Competitividad (MINECO) cofunded by the FEDER Program of EU (RN: RTI2018-099413B-I00; CD: BFU2017-87721-P; ML: RTI2018-101840-B-I00); Atresmedia Corporación (RN and ML); Fundación BBVA (RN); "la Caixa" Foundation (ID 100010434), under the agreements LCF/PR/HR19/52160016 (RN) and LCF/PR/HR19/ 52160022 (ML); European Foundation for the Study of Diabetes (RN), ERC Synergy Grant-2019-WATCH- 810331 (RN) and Western Norway Regional Health Authority (Helse Vest RHF) (JF). AF received a fellowship from Xunta de Galicia (Plan I2C2014). The CiMUS is supported by the Xunta de Galicia (20162019, ED431G/05). CIBER de Fisiopatología de la Obesidad y Nutrición is an initiative of ISCIII. The funders had no role in study design, data collection and analysis, decision to publish, or preparation of the manuscript.

\section{SUPPLEMENTARY MATERIAL}

The Supplementary Material for this article can be found online at: https://www.frontiersin.org/articles/10.3389/fendo.2021. 669980/full\#supplementary-material

10. Cerrato M, Carrera O, Vazquez R, Echevarria E, Gutierrez E. Heat Makes a Difference in Activity-Based Anorexia: A Translational Approach to Treatment Development in Anorexia Nervosa. Int J Eat Disord (2012) 45 (1):26-35. doi: 10.1002/eat.20884

11. Gutierrez E, Cerrato M, Carrera O, Vazquez R. Heat Reversal of ActivityBased Anorexia: Implications for the Treatment of Anorexia Nervosa. Int J Eat Disord (2008) 41(7):594-601. doi: 10.1002/eat.20535

12. Gutierrez E, Churruca I, Zarate J, Carrera O, Portillo MP, Cerrato M, et al. High Ambient Temperature Reverses Hypothalamic MC4 Receptor Overexpression in an Animal Model of Anorexia Nervosa. Psychoneuroendocrinology (2009) 34 (3):420-9. doi: 10.1016/j.psyneuen.2008.10.003

13. Roura I, Fraga A, Gutierrez E. Differential Effects of Heat in the Phases of the Light-Dark Cycle in the Activity-Based Anorexia Model. Int J Eat Disord (2020) 53(11):1826-35. doi: 10.1002/eat.23363

14. Hillebrand JJ, de Rijke CE, Brakkee JH, Kas MJ, Adan RA. Voluntary Access to a Warm Plate Reduces Hyperactivity in Activity-Based Anorexia. Physiol Behav (2005) 85(2):151-7. doi: 10.1016/j.physbeh.2005.03.017

15. Carrera O, Gutierrez E. Hyperactivity in Anorexia Nervosa: to Warm or Not to Warm. That is the Question (a Translational Research One). J Eat Disord (2018) 6:4. doi: 10.1186/s40337-018-0190-6

16. Fraga A, Carreira MC, Gonzalez-Izquierdo A, Dieguez C, Lopez M, Gutierrez E. Temperature But Not Leptin Prevents Semi-Starvation Induced Hyperactivity in Rats: Implications for Anorexia Nervosa Treatment. Sci Rep (2020) 10(1):5300. doi: 10.1038/s41598-020-62147-z

17. Duncan L, Yilmaz Z, Gaspar H, Walters R, Goldstein J, Anttila V, et al. Significant Locus and Metabolic Genetic Correlations Revealed in GenomeWide Association Study of Anorexia Nervosa. Am J Psychiatry (2017) 174 (9):850-8. doi: 10.1176/appi.ajp.2017.16121402 
18. Kim SF. Animal Models of Eating Disorders. Neuroscience (2012) 211:2-12. doi: 10.1016/j.neuroscience.2012.03.024

19. Claret M, Smith MA, Batterham RL, Selman C, Choudhury AI, Fryer LG, et al. AMPK is Essential for Energy Homeostasis Regulation and Glucose Sensing by POMC and AgRP Neurons. J Clin Invest (2007) 117(8):2325-36. doi: $10.1172 /$ JCI31516

20. Schneeberger M, Claret M. Recent Insights Into the Role of Hypothalamic Ampk Signaling Cascade Upon Metabolic Control. Front Neurosci (2012) 6:185. doi: 10.3389/fnins.2012.00185

21. Lopez M, Nogueiras R, Tena-Sempere M, Dieguez C. Hypothalamic AMPK: A Canonical Regulator of Whole-Body Energy Balance. Nat Rev Endocrinol (2016) 12(7):421-32. doi: 10.1038/nrendo.2016.67

22. Lopez M. Ampk Wars: The VMH Strikes Back, Return of the PVH. Trends Endocrinol Metab (2018) 29(3):135-7. doi: 10.1016/j.tem.2018.01.004

23. Lopez M, Varela L, Vazquez MJ, Rodriguez-Cuenca S, Gonzalez CR, Velagapudi VR, et al. Hypothalamic AMPK and Fatty Acid Metabolism Mediate Thyroid Regulation of Energy Balance. Nat Med (2010) 16(9):10018. doi: $10.1038 / \mathrm{nm} .2207$

24. Contreras C, Gonzalez-Garcia I, Martinez-Sanchez N, Seoane-Collazo P, Jacas J, Morgan DA, et al. Central Ceramide-Induced Hypothalamic Lipotoxicity and ER Stress Regulate Energy Balance. Cell Rep (2014) 9(1):366-77. doi: 10.1016/..celrep.2014.08.057

25. Contreras C, Gonzalez-Garcia I, Seoane-Collazo P, Martinez-Sanchez N, Linares-Pose L, Rial-Pensado E, et al. Reduction of Hypothalamic Endoplasmic Reticulum Stress Activates Browning of White Fat and Ameliorates Obesity. Diabetes (2017) 66(1):87-99. doi: 10.2337/db15-1547

26. Gonzalez-Garcia I, Contreras C, Estevez-Salguero A, Ruiz-Pino F, Colsh B, Pensado I, et al. Estradiol Regulates Energy Balance by Ameliorating Hypothalamic Ceramide-Induced Er Stress. Cell Rep (2018) 25(2):41323.e5. doi: 10.1016/j.celrep.2018.09.038

27. Martinez de Morentin PB, Gonzalez-Garcia I, Martins L, Lage R, FernandezMallo D, Martinez-Sanchez N, et al. Estradiol Regulates Brown Adipose Tissue Thermogenesis Via Hypothalamic Ampk. Cell Metab (2014) 20(1):4153. doi: 10.1016/j.cmet.2014.03.031

28. Martinez-Sanchez N, Seoane-Collazo P, Contreras C, Varela L, Villarroya J, Rial-Pensado E, et al. Hypothalamic AMPK-ER Stress-Jnk1 Axis Mediates the Central Actions of Thyroid Hormones on Energy Balance. Cell Metab (2017) 26(1):212-29.e12. doi: 10.1016/j.cmet.2017.06.014

29. Seoane-Collazo P, Linares-Pose L, Rial-Pensado E, Romero-Pico A, MorenoNavarrete JM, Martinez-Sanchez N, et al. Central Nicotine Induces Browning Through Hypothalamic Kappa Opioid Receptor. Nat Commun (2019) 10 (1):4037. doi: 10.1038/s41467-019-12004-z

30. Martins L, Seoane-Collazo P, Contreras C, Gonzalez-Garcia I, MartinezSanchez N, Gonzalez F, et al. A Functional Link Between AMPK and Orexin Mediates the Effect of BMP8B on Energy Balance. Cell Rep (2016) 16(8):223142. doi: 10.1016/j.celrep.2016.07.045

31. Seoane-Collazo P, Romero-Pico A, Rial-Pensado E, Linares-Pose L, EstevezSalguero A, Ferno J, et al. Kappa-Opioid Signaling in the Lateral Hypothalamic Area Modulates Nicotine-Induced Negative Energy Balance. Int J Mol Sci (2021) 22(4):1515. doi: 10.3390/ijms22041515

32. Martinez-Sanchez N, Moreno-Navarrete JM, Contreras C, Rial-Pensado E, Ferno J, Nogueiras R, et al. Thyroid Hormones Induce Browning of White Fat. J Endocrinol (2017) 232(2):351-62. doi: 10.1530/JOE-16-0425

33. Haas V, Onur S, Paul T, Nutzinger DO, Bosy-Westphal A, Hauer M, et al. Leptin and Body Weight Regulation in Patients With Anorexia Nervosa Before and During Weight Recovery. Am J Clin Nutr (2005) 81(4):889-96. doi: 10.1093/ajen/81.4.889

34. Focker M, Timmesfeld N, Scherag S, Buhren K, Langkamp M, Dempfle A, et al. Screening for Anorexia Nervosa Via Measurement of Serum Leptin Levels. J Neural Transm (Vienna) (2011) 118(4):571-8. doi: 10.1007/s00702010-0551-z

35. Haas VK, Gaskin KJ, Kohn MR, Clarke SD, Muller MJ. Different Thermic Effects of Leptin in Adolescent Females With Varying Body Fat Content. Clin Nutr (2010) 29(5):639-45. doi: 10.1016/j.clnu.2010.03.013

36. Korek E, Krauss H, Gibas-Dorna M, Kupsz J, Piatek M, Piatek J. Fasting and Postprandial Levels of Ghrelin, Leptin and Insulin in Lean, Obese and Anorexic Subjects. Prz Gastroenterol (2013) 8(6):383-9. doi: 10.5114/ pg.2013.39922
37. Misra M, Miller KK, Kuo K, Griffin K, Stewart V, Hunter E, et al. Secretory Dynamics of Leptin in Adolescent Girls With Anorexia Nervosa and Healthy Adolescents. Am J Physiol Endocrinol Metab (2005) 289(3):E373-81. doi: 10.1152/ajpendo.00041.2005

38. Grinspoon S, Gulick T, Askari H, Landt M, Lee K, Anderson E, et al. Serum Leptin Levels in Women With Anorexia Nervosa. J Clin Endocrinol Metab (1996) 81(11):3861-3. doi: 10.1210/jcem.81.11.8923829

39. Hebebrand J, Exner C, Hebebrand K, Holtkamp C, Casper RC, Remschmidt $\mathrm{H}$, et al. Hyperactivity in Patients With Anorexia Nervosa and in Semistarved Rats: Evidence for a Pivotal Role of Hypoleptinemia. Physiol Behav (2003) 79 (1):25-37. doi: 10.1016/S0031-9384(03)00102-1

40. Hotta M, Shibasaki T, Masuda A, Imaki T, Demura H, Ling N, et al. The Responses of Plasma Adrenocorticotropin and Cortisol to CorticotropinReleasing Hormone (CRH) and Cerebrospinal Fluid Immunoreactive CRH in Anorexia Nervosa Patients. J Clin Endocrinol Metab (1986) 62(2):319-24. doi: 10.1210/jcem-62-2-319

41. Kaye WH, Gwirtsman HE, George DT, Ebert MH, Jimerson DC, Tomai TP, et al. Elevated Cerebrospinal Fluid Levels of Immunoreactive CorticotropinReleasing Hormone in Anorexia Nervosa: Relation to State of Nutrition, Adrenal Function, and Intensity of Depression. J Clin Endocrinol Metab (1987) 64(2):203-8. doi: 10.1210/jcem-64-2-203

42. Lo Sauro C, Ravaldi C, Cabras PL, Faravelli C, Ricca V. Stress, HypothalamicPituitary-Adrenal Axis and Eating Disorders. Neuropsychobiology (2008) 57 (3):95-115. doi: 10.1159/000138912

43. de Rijke CE, Hillebrand JJ, Verhagen LA, Roeling TA, Adan RA. Hypothalamic Neuropeptide Expression Following Chronic Food Restriction in Sedentary and Wheel-Running Rats. J Mol Endocrinol (2005) 35(2):381-90. doi: 10.1677/jme.1.01808

44. Burden VR, White BD, Dean RG, Martin RJ. Activity of the HypothalamicPituitary-Adrenal Axis is Elevated in Rats With Activity-Based Anorexia. J Nutr (1993) 123(7):1217-25. doi: 10.1093/jn/123.7.1217

45. Sentis SC, Oelkrug R, Mittag J. Thyroid Hormones in the Regulation of Brown Adipose Tissue Thermogenesis. Endocr Connect. (2021) 10(2):R106-15. doi: 10.1530/EC-20-0562

46. Lopez M, Alvarez CV, Nogueiras R, Dieguez C. Energy Balance Regulation by Thyroid Hormones At Central Level. Trends Mol Med (2013) 19(7):418-27. doi: 10.1016/j.molmed.2013.04.004

47. Bredella MA, Ghomi RH, Thomas BJ, Torriani M, Brick DJ, Gerweck AV, et al. Comparison of DXA and CT in the Assessment of Body Composition in Premenopausal Women With Obesity and Anorexia Nervosa. Obes (Silver Spring) (2010) 18(11):2227-33. doi: 10.1038/oby.2010.5

48. Misra M, Soyka LA, Miller KK, Grinspoon S, Levitsky LL, Klibanski A. Regional Body Composition in Adolescents With Anorexia Nervosa and Changes With Weight Recovery. Am J Clin Nutr (2003) 77(6):1361-7. doi: 10.1093/ajcn/77.6.1361

49. Grinspoon S, Thomas E, Pitts S, Gross E, Mickley D, Miller K, et al. Prevalence and Predictive Factors for Regional Osteopenia in Women With Anorexia Nervosa. Ann Intern Med (2000) 133(10):790-4. doi: 10.7326/0003-4819-13310-200011210-00011

50. Bredella MA, Fazeli PK, Freedman LM, Calder G, Lee H, Rosen CJ, et al. Young Women With Cold-Activated Brown Adipose Tissue Have Higher Bone Mineral Density and Lower Pref-1 Than Women Without Brown Adipose Tissue: A Study in Women With Anorexia Nervosa, Women Recovered From Anorexia Nervosa, and Normal-Weight Women. J Clin Endocrinol Metab (2012) 97(4):E584-90. doi: 10.1210/jc.2011-2246

51. Nedergaard J, Cannon B. The Browning of White Adipose Tissue: Some Burning Issues. Cell Metab (2014) 20(3):396-407. doi: 10.1016/j.cmet. 2014.07.005

52. Cereijo R, Giralt M, Villarroya F. Thermogenic Brown and Beige/Brite Adipogenesis in Humans. Ann Med (2015) 47(2):169-77. doi: 10.3109/ 07853890.2014 .952328

53. Villarroya F, Cereijo R, Villarroya J, Gavalda-Navarro A, Giralt M. Toward an Understanding of How Immune Cells Control Brown and Beige Adipobiology. Cell Metab (2018) 27(5):954-61. doi: 10.1016/j.cmet.2018.04.006

54. Shabalina IG, Petrovic N, de Jong JM, Kalinovich AV, Cannon B, Nedergaard J. UCP1 in Brite/Beige Adipose Tissue Mitochondria is Functionally Thermogenic. Cell Rep (2013) 5(5):1196-203. doi: 10.1016/j.celrep. 2013.10.044 
55. Petruzzelli M, Schweiger M, Schreiber R, Campos-Olivas R, Tsoli M, Allen J, et al. A Switch From White to Brown Fat Increases Energy Expenditure in Cancer-Associated Cachexia. Cell Metab (2014) 20(3):433-47. doi: 10.1016/ j.cmet.2014.06.011

56. Petruzzelli M, Wagner EF. Mechanisms of Metabolic Dysfunction in CancerAssociated Cachexia. Genes Dev (2016) 30(5):489-501. doi: 10.1101/ gad.276733.115

57. Lipsman N, Woodside DB, Lozano AM. Neurocircuitry of Limbic Dysfunction in Anorexia Nervosa. Cortex (2015) 62:109-18. doi: 10.1016/ j.cortex.2014.02.020

58. Gutierrez E, Baysari MT, Carrera O, Whitford TJ, Boakes RA. High Ambient Temperature Reduces Rate of Body-Weight Loss Produced by Wheel Running. Q J Exp Psychol (Hove). (2006) 59(7):1196-211. doi: 10.1080/ 17470210500417688

59. Cannon B, Nedergaard J. Brown Adipose Tissue: Function and Physiological Significance. Physiol Rev (2004) 84(1):277-359. doi: 10.1152/physrev. 00015.2003

60. Oelkrug R, Mittag J. An Improved Method for the Precise Unravelment of non-Shivering Brown Fat Thermokinetics. Sci Rep (2021) 11(1):4799. doi: 10.1038/s41598-021-84200-1

61. Sakurada S, Shido O, Sugimoto N, Hiratsuka Y, Yoda T, Kanosue K. Autonomic and Behavioural Thermoregulation in Starved Rats. J Physiol (2000) 526(Pt 2):417-24. doi: 10.1111/j.1469-7793.2000.00417.x
62. Young JB, Saville E, Rothwell NJ, Stock MJ, Landsberg L. Effect of Diet and Cold Exposure on Norepinephrine Turnover in Brown Adipose Tissue of the Rat. J Clin Invest (1982) 69(5):1061-71. doi: 10.1172/JCI110541

63. Zhang LN, Mitchell SE, Hambly C, Morgan DG, Clapham JC, Speakman JR Physiological and Behavioral Responses to Intermittent Starvation in C57BL/ 6J Mice. Physiol Behav (2012) 105(2):376-87. doi: 10.1016/j.physbeh. 2011.08.035

64. Nobis S, Goichon A, Achamrah N, Guerin C, Azhar S, Chan P, et al. Alterations of Proteome, Mitochondrial Dynamic and Autophagy in the Hypothalamus During Activity-Based Anorexia. Sci Rep (2018) 8(1):7233. doi: 10.1038/s41598-018-25548-9

Conflict of Interest: The authors declare that the research was conducted in the absence of any commercial or financial relationships that could be construed as a potential conflict of interest.

Copyright (c) 2021 Fraga, Rial-Pensado, Nogueiras, Fernø, Diéguez, Gutierrez and Lopez. This is an open-access article distributed under the terms of the Creative Commons Attribution License (CC BY). The use, distribution or reproduction in other forums is permitted, provided the original author(s) and the copyright owner(s) are credited and that the original publication in this journal is cited, in accordance with accepted academic practice. No use, distribution or reproduction is permitted which does not comply with these terms. 